\title{
Surface Property Tuning of Methylammonium Lead lodide by Plasma for Use in Planar Perovskite Solar Cells
}

\author{
Masoud Shekargoftar,* Jan Pospisil, Ján Dugáček, Martin Weiter, and Tomáš Homola \\ Cite This: ACS Omega 2020, 5, 18384-18390 \\ Read Online
}

ABSTRACT: The demand for cheap and green energy as a replacement for fossil fuels has never been greater, and perovskite solar cells (PSCs) are among the leading means of meeting it. The surface properties of metal halide perovskite layers play crucial roles in the performance and durability of such cells. Consequently, a wide range of engineering processes for surface modification of perovskite layers has been investigated and among them is atmospheric pressure plasma (APP). Nevertheless, knowledge of the interaction between plasma and perovskite layers is still far from complete. In this work, $\mathrm{CH}_{3} \mathrm{NH}_{3} \mathrm{PbI}_{3}$ films were subjected to APP generated by a portable plasma source. A detailed understanding of band energy after plasma treatment is

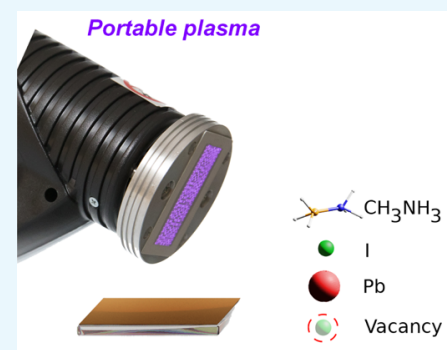

Plasma treatment of perovskite layer

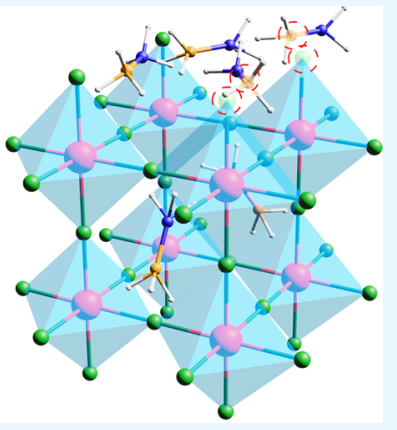
crucial to the investigation of the behavior of the perovskite layer. This study demonstrates a remarkable shift in the valence and conduction bands of a perovskite layer after plasma treatment, while band gap energy remains relatively constant. We found that short plasma treatment of perovskite layers resulted in higher performance and stability of PSCs.

\section{INTRODUCTION}

Perovskite solar cells (PSCs) have become the focus of enormous interest in recent years; their power conversion efficiency (PCE) is high and their costs of fabrication are low. ${ }^{1}$ Within a decade, the PCE of PSCs has surpassed $25.2 \%$ in response to a wide range of procedures and among them are compositional engineering, interface engineering, and perovskite film growth control. ${ }^{2-5}$ The outstanding performance of PSCs is mainly related to the electronic band structure of the active perovskite layer. Metal halide perovskite materials exhibit an extended diffusion length and a strong absorption coefficient, originating out of their favorable direct $p-p$ transition band gap. ${ }^{6,7}$ However, these excellent optoelectrical properties are accompanied by grain boundaries and a high density of defects on the surface of any solution-processed perovskite layer. Although considerable efforts have been devoted to understanding the physics of perovskite materials, surface engineering technologies have been in quest of large and high-quality perovskite layers. The surface morphology and chemical composition of perovskite films are generally agreed to be among the primary parameters of a significant impact on the performance of PSCs; ${ }^{8}$ several surface modification techniques have been tested with a view to optimize the surface properties of perovskite films, including vacuum flash-assisted, ${ }^{9}$ gas-assisted, ${ }^{10}$ and, very recently, plasma-assisted solution processes. $^{11}$ Some of the surface treatment techniques could passivate undesirable defects, while others tune the morphology of the perovskite films. For instance, Tavakoli and co-workers ${ }^{12,13}$ employed adamantane additives to mitigate the electronic defects at the interface of perovskite and the hole transport layer (HTL). They found that this technique improved the performance and the stability of the PSCs. Si et al. ${ }^{14}$ reported passivation of defects by incorporating ammonium chloride. $\mathrm{Wu}$ and co-workers ${ }^{15}$ introduced a technique called "multiple ligands" to passivate surface defects of perovskite films which increased the PCE of PSCs.

Recently, focus has come to shift onto the effects of various plasma technologies on the properties of halide perovskite $\left(\mathrm{CH}_{3} \mathrm{NH}_{3} \mathrm{PbI}_{3}\right)$. Plasma has attracted increasing attention because of its unique capability to modify materials under a wide range of conditions. The plasma-surface interactions and flux of the species transferring from plasma to the exposed surface are largely determined by sheath voltage, power density, and the pressure of the plasma. In terms of pressure, plasma may be classified into low and high. Low-pressure plasma (generated at a pressure of $\leq 10^{3} \mathrm{~Pa}$ ) has found widespread application in the microelectronics industry. Very recently, Huang et al. have reported low-pressure Ar plasma treatment of a perovskite (PTPV) layer before the deposition

Received: May 11, 2020

Accepted: July 1, 2020

Published: July 16, 2020 

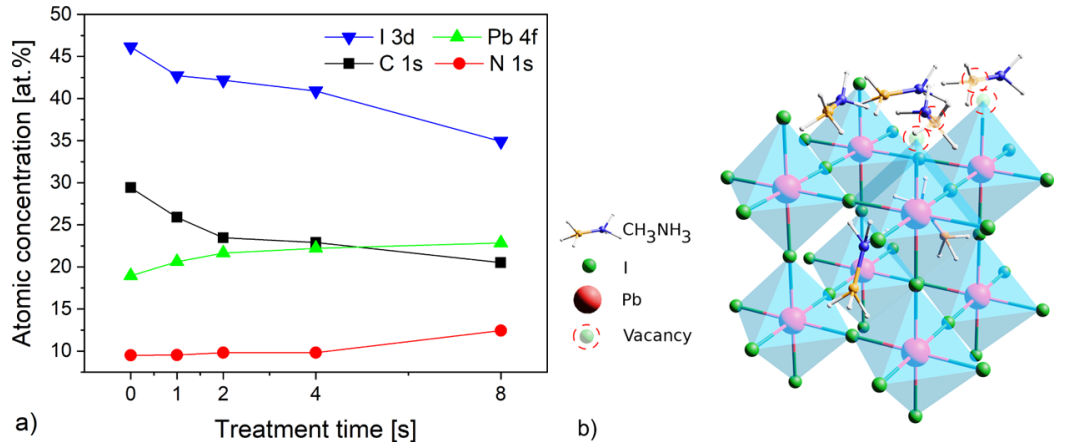

Figure 1. (a) Atomic concentration determined from XPS spectra and (b) possible mechanism for induced changes on the perovskite surface after exposure to plasma.

of a fullerene passivating layer. ${ }^{11}$ Ar plasma tuned the ratio between organic and inorganic compounds of $\mathrm{CH}_{3} \mathrm{NH}_{3} \mathrm{PbI}_{3}$, enhancing charge collection across the perovskite-electrode interface by suppressing charge recombination. However, lowpressure plasma treatment requires a vacuum chamber, and pumping involved in achieving this state results in increased manufacturing costs. In this light, researchers have turned to attempts at plasma treatment at atmospheric pressure"atmospheric-pressure plasma (APP)". The latter plasma has been used extensively in the fabrication of solar cells. For example, different types of APP have been introduced for the surface decontamination of FTO and ITO surfaces. ${ }^{16,17}$ APP treatments have been introduced to modify the surface properties of $\mathrm{CH}_{3} \mathrm{NH}_{3} \mathrm{PbI}_{3}$ films. ${ }^{18}$ This plasma treatment resulted in a lead-rich surface and passivated the surface defects of perovskite layers. The effects of APPs on the properties of perovskite layers and on the performance of PSCs have already been reported; nevertheless, they remain highly topical issues. Generally, plasma contains highly reactive species and is likely to react with the lattice environment of organic methylammonium species, interstitial iodide ions, and subsurface lead. Despite such previous reports, no mechanism for the plasmainduced changes on the perovskite surface has yet been proposed.

The high-energy electron in plasma causes ionization, excitation, oxidation, and fragmentation of molecules of working gas, which makes plasma chemically active. Plasma contains various species such as positive/negative ions, free radicals, neutral atoms, and metastable species, and exposure of the surface to these species during plasma modification led to different processes. ${ }^{19}$ The highly energetic reactive species bombarded the molecules on the surface and broke them into volatile and smaller molecules. The chemical properties of the working gas and exposed materials are the main parameters that determine the reaction of the plasma with the surface. In this work, APP was generated using portable plasma with a diffuse coplanar surface barrier discharge (DCSBD) plasma unit. The plasma source operated within a glovebox with a nitrogen environment. The plasma led to partial removal of the organic component and resulted in lead-enhanced perovskite layers. PTPV partially passivated surface defects prior to depositing the PCBM layer. PTPV led to enlarged grain size and involved negligible etching $(\sim 2 \mathrm{~nm})$. Surprisingly, the intensity of photoluminescence (PL) dropped after plasma treatment. UV-vis spectroscopy and ultraviolet photoelectron spectroscopy (UPS) were employed to investigate the mechanism, leading to the drop in PL. Plasma treatment had resulted in a shift in the electronic band of the perovskite films.
PSCs were then fabricated with an inverted structure, and PTPV led to a significant improvement in the photovoltaic parameters of the PSCs.

\section{RESULTS AND DISCUSSION}

Surface Properties of Perovskite Films. X-ray photoelectron spectroscopy (XPS) measurements were taken to evaluate the PTPV films. The concentrations of C $1 \mathrm{~s}, \mathrm{~N} 1 \mathrm{~s}, \mathrm{I}$ $3 \mathrm{~d}$, and $\mathrm{Pb} 4 \mathrm{f}$ appear in Figure 1a. Plasma treatment led to partial removal of the organic component from the surface because a significant decrease in C 1 s was observed after plasma treatment. Furthermore, plasma treatment lowered the concentration of I 3d. Although the carbon concentration decreased, the concentration of $\mathrm{Pb} 4 \mathrm{f}$ increased after PTPV. The estimated $\mathrm{Pb} / \mathrm{I}$ ratio for the untreated perovskite film was $1: 2.43$, reaching $1: 1.86$ after $1 \mathrm{~s}$ plasma treatment of the surface. Plasma treatment times of $2 \mathrm{~s}$ and $4 \mathrm{~s}$ resulted in $\mathrm{Pb} / \mathrm{I}$ ratios of $1: 1.84$ and $1: 1.71$, respectively, and the $\mathrm{Pb} / \mathrm{I}$ ratio reached 1:1.67 after plasma treatment for $8 \mathrm{~s}$. Such increases in $\mathrm{Pb} 4 \mathrm{f}$ could be explained by removal of the organic component from the surface. The $\mathrm{Pb}$ components were covered by the organic component, and thus, removing the organic part resulted in an increase in $\mathrm{Pb}$ and the formation of a leadenhanced surface. The high-resolution XPS spectra appear in Figure S1 (Supporting Information). It is seen that a peak at $286.4 \mathrm{eV}$, corresponding to $\mathrm{C}-\mathrm{O}$ bonds, and a peak at 137.7 $\mathrm{eV}$, corresponding to $\mathrm{Pb}=\mathrm{O}$ bonds, decreased significantly after treatment, suggesting that such plasma may slow the degradation of the perovskite layer by suppressing oxygen moisture formation on the surface. Because plasma treatment was performed in a nitrogen environment, the concentration of $\mathrm{N}$ 1s was enhanced after the plasma treatment.

Mechanism of Plasma-Induced Defects. As is seen from Figure $1 \mathrm{~b}$, decreases in $\mathrm{C} 1 \mathrm{~s}$ and I $3 \mathrm{~d}$ peaks on the surface led to point defects. These defects could well lead to shallow donor or acceptor levels. Taking into account the Schottky defects for the $\mathrm{CH}_{3} \mathrm{NH}_{3} \mathrm{PbI}_{3}$ and following the notation of Kröger and Vink ${ }^{20}$

$$
\text { nil } \rightarrow V_{\mathrm{MA}}^{\prime}+V_{\mathrm{Pb}}^{\prime \prime}+3 V_{\mathrm{I}}^{\bullet}
$$

where nil represents perfect $\mathrm{CH}_{3} \mathrm{NH}_{3} \mathrm{PbI}_{3}, V$ represents a vacancy, and subscripts represent the corresponding ions. The vacancies appear as superscripts, with effective charges, with a dot for positive and a prime for each negative charge. ${ }^{21}$ According to Walsh et al., the concentration of $\mathrm{MA}^{+}, \mathrm{Pb}^{2+}$, and $\mathrm{I}^{-}$vacancies is in equilibrium under room conditions. ${ }^{20}$ Plasma contains energetic species such as electrons, ions, metastable 

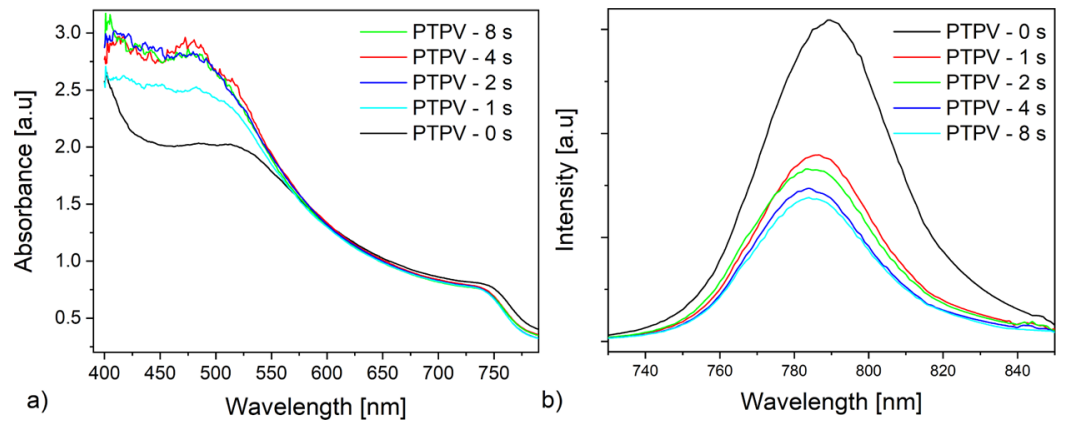

Figure 2. (a) Absorbance and (b) steady-state PL spectra of lead iodide perovskite with various plasma treatment times.

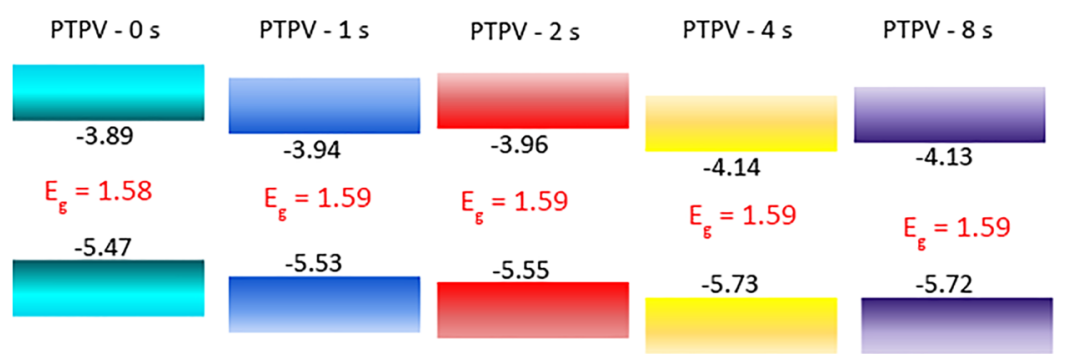

Figure 3. Energy band diagram of lead iodide perovskite films for various plasma treatment times.

species, and neutral particles capable of breaking the bonds and changing the concentration equilibrium of the abovementioned vacancies.

Scanning electron microscopy (SEM) measurements were carried out on the PTPV films to evaluate their morphological behavior. As is evident from the SEM images, Figure S2, plasma treatment led to the enlargement of grain size on the surface of the perovskite films. The results correlate well with the work of Tsai et al., in which plasma treatment led to an increase in grain size in $\mathrm{CH}_{3} \mathrm{NH}_{3} \mathrm{PbI}_{3}$ perovskite films. ${ }^{18}$ In addition, the measured thickness of the PTPV films (Figure S3) shows a slight difference before and after plasma treatment $(1-5 \mathrm{~nm})$. These superficial effects are to be anticipated because APP has a very low mean free path, in the order of tens of nanometers, ${ }^{22}$ and the coplanar configuration of the plasma source resulted in highly diffuse plasma. The results indicated a very low etching effect upon the perovskite films after plasma treatment, probably originating in the removal of organic components. In contrast, ambient air plasma treatment for $5 \mathrm{~s}$ etched away more than $18 \mathrm{~nm}$ of the perovskite films. ${ }^{23}$ The effects of plasma on perovskite films were further investigated with X-ray diffraction (XRD) (Figure S4). The presence of (220), (310), (224), and (330) can be indexed to the $\mathrm{CH}_{3} \mathrm{NH}_{3} \mathrm{PbI}_{3} \cdot{ }^{24}$ No considerable changes were observed on the peak at $28.1^{\circ}$, but plasma treatment slightly increased the intensity of the peaks at 34.9 and $44.2^{\circ}$. The intensity of the peak at $31.5^{\circ}$ enhanced after plasma treatment. This peak was assigned to metallic lead, ${ }^{11}$ and its enhancement is in agreement with XPS data as plasma treatment led to a lead-rich perovskite film.

Optoelectronic Properties of Perovskite Films. Plasma-induced changes in the optical properties of the perovskite films were investigated by means of steady-state PL and UVvis. The absorption spectra of the various PTPV films appear in Figure $2 \mathrm{a}$. The enhanced absorbance at short wavelengths after plasma treatment may be due to changes in the $\mathrm{Pb} / \mathrm{I}$ ratio and improved surface homogeneity after plasma treatment. ${ }^{25}$ Figure $2 \mathrm{~b}$ presents a comparison of the steady-state PL spectra of the perovskite films. The PL maximum and band edge of absorbance spectra shift toward lower wavelengths after plasma treatment, exhibiting a widening of the optical band gap. The shifted PL spectra could be related to the passivation of the trap states on the surface after plasma treatment. ${ }^{26}$ The calculated band gap of the films appears in Figure 3. However, a progressive decrease in PL intensity becomes evident after plasma treatment, possibly a response to better electron extraction and a more homogeneous perovskite surface ${ }^{27,28}$ or perhaps originating in shifted energy levels within the perovskite after plasma treatment. UPS measurements were taken to gain further insight into the effects of plasma on the energy levels of the perovskite films. As is evident from the UPS spectra (Figure S5), plasma treatment times of 1 and $2 \mathrm{~s}$ shifted the secondary electron (SE) cutoff toward higher binding energy, resulting in a lower work function for the perovskite films. The work function was calculated by subtracting binding energy at the SE cutoff (energy of the slowest emitted electron) from the energy of the incident light $(21.22 \mathrm{eV})$. The details of UPS measurement and calculation of the work function can be found in ref 29. In addition, the valence band maximum (VBM) can be determined from the UPS spectra with respect to the calculated band gap energy (from absorbance spectra). The calculated work function of the untreated perovskite film was $\sim 4.53 \mathrm{eV}$, falling to 4.48 and $4.45 \mathrm{eV}$ after 1 and $2 \mathrm{~s}$ of plasma treatment, respectively. Longer plasma treatment times increased the work function, extending it to as much as $4.72 \mathrm{eV}$ after a treatment time of $8 \mathrm{~s}$. As Figure 3 demonstrates, VBM and conduction band minimum (CBM) shifted slightly to higher energy after plasma treatment. In principle, VBM and CBM of halide perovskite $\mathrm{ABX}_{3}$ may be tuned by changes in the chemical nature of $\mathrm{A}, \mathrm{B}$, and $\mathrm{X}^{30}$ According to density functional theory calculation, as reported by Meloni et al., VBM and CBM arise out of the antibonding combination of $\mathrm{Pb}$ and $\mathrm{I}^{31}$ It appears, therefore, that changing the concentrations of $\mathrm{Pb}$ and I led to the changes in VBM and CBM levels. 

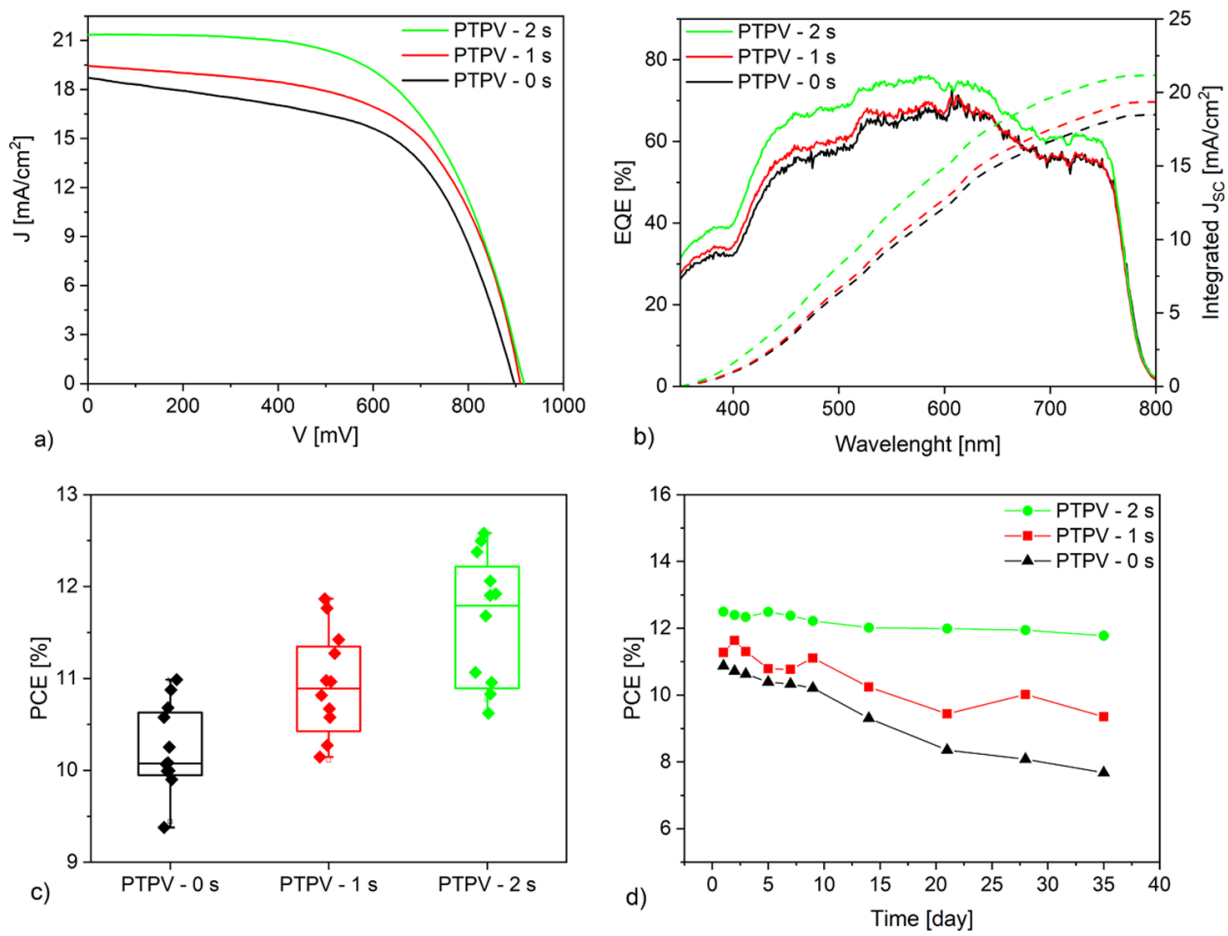

Figure 4. Performance parameters of PSCs with various PTPV layers: (a) current density-voltage $(J-V)$ curves, (b) EQE with the corresponding integrated current density, (c) statistical distribution of PCE, and (d) comparison of performance stability for (champions) PSCs with plasmatreated perovskite layers.

Effects of Plasma on the Performance of PSCs. Inverted PSCs were fabricated to evaluate the practical functionality of PTPV films. Furthermore, the effects of plasma treatment on hysteresis behavior were evaluated by calculating the hysteresis index. The details of the calculation are presented in the Supporting Information. The hysteresis index values for one of the measurements are listed in Table S1. The PCE values were taken from devices with photovoltaic parameters close to average values. Figure $4 a$ illustrates the current density versus voltage $(J-V)$ curves of PSCs with PTPV films. The measurements were taken immediately after device fabrication. The device with an untreated perovskite layer (PTPV-0 s) showed an efficiency of $10.25 \%$ with a fill factor (FF) of $60.89 \%$, an open-circuit voltage of $897.24 \mathrm{mV}$, and a short current density of $18.73 \mathrm{~mA} / \mathrm{cm}^{2}$. The performance of the PSCs varied with plasma treatment time. As is evident from the $J-V$ curve, PTPV-2 $\mathrm{s}$ resulted in higher device efficiency with a $J_{\mathrm{SC}}$ of $21.52 \mathrm{~mA} / \mathrm{cm}^{2}$, a $V_{\mathrm{OC}}$ of 914.04 $\mathrm{mV}$, an FF of $59.67 \%$, and a PCE of $11.68 \%$. The summary of the photovoltaic performance for all devices is presented in Table S2. Because longer treatment time starts to deteriorate the PSC performance, therefore, only PTPV-1 s and PTPV- $2 \mathrm{~s}$ were selected for further evaluation. It was observed that the PTPV layer for a shorter time led to increasing short current density of the device. The results confirmed by integrated short current density measurement are presented in Figure $4 \mathrm{~b}$. The current density improvement could be attributed to the fact that plasma treatment may have effectively removed carbon concentrations from the surface and thus enhanced electrical conductivity. As was observed from the XPS results, plasma treatment decreases the concentration of organic components on the surface. This alone might improve the conductivity of the perovskite layer that resulted in the higher current density. As is evident in Figure $4 b$, the highest external quantum efficiency (EQE) was observed for the device with the PTPV-2 s film, meaning improved carrier collection. Figure $4 \mathrm{c}$ shows the statistics of photovoltaic characteristics of the PSCs with different perovskite layers. Short plasma treatment enhanced the performance of the PSCs mainly because of improving $J_{\mathrm{SC}}$. In addition, the PTPV layer improved $V_{\mathrm{OC}}$ of the PSCs. Carrier recombination in PSCs has a considerable impact on open-circuit voltage. It was observed that plasma increased $V_{\mathrm{OC}}$, something that may be explained by carrier recombination at the interfaces and effects of the plasma on the band energy alignment of the perovskite. It has been shown that recombination at the perovskite/electron transport layer and $\mathrm{HTL} /$ perovskite interfaces could significantly influence the electrical performance of the device. ${ }^{32}$ It might be suggested that plasma treatment resulted in a lead-rich surface that could well increase the interaction between perovskite and PCBM to reduce charge recombination. Furthermore, as observed in the previous section, PTPV led to a shift in the VBM and CBM levels of the perovskite layer, which could explain the higher $V_{\mathrm{OC}}$ of the device with plasma-treated perovskite films. It should be noted that the external quantum efficiencies of the devices changed in response to measured current density. The samples with the highest efficiencies were selected for evaluating long-time stability. Figure $4 \mathrm{~d}$ shows that PSCs with plasma-treated perovskite layers showed improved longterm stability. PTPV layers before the deposition of PCBM leads to an enhancement in the passivation of the defect state and better charge mobility which contributed to the improvement of performance and long-term stability of the PSCs. The stability of the device with PTPV-2 s was significantly higher than that of other devices where it could retain more than $94 \%$ of its initial PCE value after 35 days under standard conditions. 

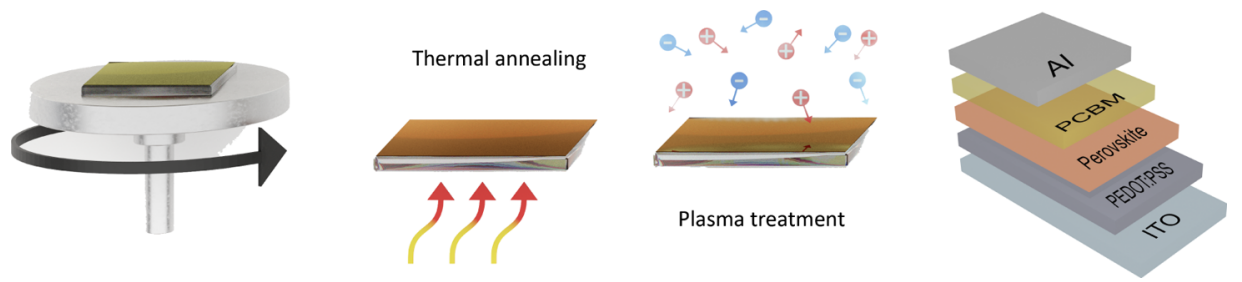

Figure 5. Illustration of the plasma-assisted fabrication of the solution-processed methylammonium lead iodide film to be used in an invertedstructure PSC.

\section{CONCLUSIONS}

Here, plasma treatment of lead iodide perovskite was demonstrated as a promising technique for enhancing the efficiency of solar cells. In this work, a comprehensive investigation was conducted on the effects of APP on the surface properties of a lead halide perovskite layer and subsequently on the performance of PSCs. Perovskite layers were prepared over PEDOT/PSS and laterally treated by plasma before deposition via the PCBM passivating layer. We proposed a mechanism for the plasma-induced changes over the surface. Furthermore, it was demonstrated that energetic species in plasma can tune the energy band of lead iodide perovskite by changing the surface chemistry and disrupting the vacancy equilibrium on the surface. Plasma treatment removes organic components and passivates defects states on the surface of the perovskite layer before deposition of PCBM. Finally, we examined the effects of plasma on the performance and stability of the PSCs. We indicated that plasma treatment can be incorporated into PSCs to enhance the $V_{\mathrm{OC}}$ to 16.77 $\mathrm{mV}$, give a $2.79 \mathrm{~mA} / \mathrm{cm}^{2}$ increase in $J_{\mathrm{SC}}$, and deliver a $1.43 \%$ increase in steady-state PCE. In addition, a device with the plasma-treated layer (PTPV-2 s) shows excellent stability with slight PCE loss after 35 days under standard conditions.

\section{MATERIALS AND METHODS}

Plasma Treatment. PTPV layers was performed with an RPS40 portable plasma source (Roplass s.r.o., Czech Republic) with a DCSBD plasma unit, operated directly in a nitrogen glovebox. The RPS40 portable plasma source appears in Figure S7 (Supporting Information), and illustration of the different steps in the plasma-assisted fabrication of perovskite films is presented in Figure 5. The discharge was generated by a highfrequency voltage $(25 \mathrm{kHz})$ with $10 \mathrm{kV}$ peak-to-peak voltage at a power density of $70 \mathrm{~W} / \mathrm{m}^{3}$. One of the main features of the RPS40 with a DCSBD plasma unit is the generation of highly diffuse plasma, which resulted in a mild treatment of the surface. The total plasma area was $25 \times 50 \mathrm{~mm}^{2}$, and the thickness of the plasma is $\sim 0.2 \mathrm{~mm}$. The temperature of the plasma unit during the treatment was approximately $60{ }^{\circ} \mathrm{C} .{ }^{33,34}$ Treatment times were $1,2,4$, and $8 \mathrm{~s}$, and samples were ordered in terms of "PVPT-treatment time".

Material Characterization. Analysis of the samples was conducted by XPS using an ESCALAB 250Xi of an Al K $\alpha$ Xray source (ThermoFisher Scientific). XPS spectra were acquired from two spots $(650 \mu \mathrm{m})$ with a takeoff angle of $90^{\circ}$ in $10^{-8} \mathrm{mbar}$ vacuum at $20^{\circ} \mathrm{C}$. UPS was performed using a Kratos Supra under normal emission using He I (21.22 eV). The obtained spectra were calibrated at the Fermi edge, which is the energy of the fastest emitted electron. A negative bias was applied to the samples to separate the SE cutoff of the samples from that of the detector. Optical absorption spectra of the samples were measured with a Varian Cary Probe 50
UV-vis spectrophotometer. The steady-state PL spectra of the perovskite films were obtained via a FS5 spectrofluorometer from Edinburgh Instruments. XRD was conducted using a Rigaku Smartlab, $\mathrm{Cu} \mathrm{K} \alpha(\lambda=1.54059 \AA)$ radiation. A MIRA3 scanning electron microscope (Tescan, Czech Republic) was used to evaluate the morphology of the perovskite films before and after plasma treatment. The effects of plasma treatment on the thickness of perovskite films were evaluated using a mechanical profilometer (Bruker, Tucson, AZ, USA). Surface characterizations of perovskite films (XPS, UPS, PL, SEM, $\mathrm{XRD}$, and profilometry) were implemented on the perovskite films deposited on the quartz substrate.

Device Fabrication. Inverted-structure PSCs (Figure 5) were chosen to address the effect of the plasma treatment on the device performance. Chemicals (perovskite precursor, $\mathrm{PC}_{70} \mathrm{BM}$ with purity higher than $99 \%$, encapsulation epoxy) and ITO substrates were purchased from Ossila. First, patterned ITO substrates were ultrasonically cleaned with distilled water, ethanol, and isopropyl alcohol successively. For the HTL, PEDOT/PSS solution (Heraeus Clevios HC 4083) was deposited on the substrate, spun at $3000 \mathrm{rpm}$ for $30 \mathrm{~s}$, and then annealed at $120{ }^{\circ} \mathrm{C}$ on a hot plate for $30 \mathrm{~min}$. Perovskite ink was dropped onto the spinning PETOD/PSS film (50 $\mu \mathrm{L}$, $3000 \mathrm{rpm}$, in a nitrogen glovebox), and after $30 \mathrm{~s}$, the samples were placed on the hot plate $\left(80^{\circ} \mathrm{C}\right)$ and annealed for $90 \mathrm{~min}$. After the PTPV layers, $35 \mu \mathrm{L}$ of $\mathrm{PC}_{70} \mathrm{BM}$ ink $(30 \mathrm{mg}$ of $\mathrm{PC}_{70} \mathrm{BM}$ was dissolved overnight in $0.5 \mathrm{~mL}$ of chlorobenzene and $0.5 \mathrm{~mL}$ of chloroform) was spin-coated on the perovskite films at $1000 \mathrm{rpm}$. Subsequently, all the samples were moved into the evaporation chamber for deposition of the cathode. Approximately $150 \mathrm{~nm}$ of aluminum was deposited on the samples using a thermal evaporation method. Finally, all the samples were encapsulated to protect the devices from oxygen and moisture-this process is carried out using a glass coverslip and an encapsulating epoxide that is exposed to UV radiation for $15 \mathrm{~min}$.

Device Characterization. Characterization (PCE) of the device was performed under standard conditions using an AAA-certified Abet Sun solar simulator with an air mass (AM) $1.5 \mathrm{G}$ filter (the simulated light intensity was adjusted to 1000 $\mathrm{W} / \mathrm{m}^{2}$ with an NREL-calibrated Si solar cell). A shadow mask (area $0.046 \mathrm{~cm}^{2}$ ) was in place throughout the PCE measurements, EQE was assessed by means of a combined apparatus consisting of a Keithley 6478 picoammeter, an LSH502 LOT Oriel xenon lamp, an MSH101 LOT ORIEL monochromator, and a SiQE120 RaRe Solutions photometric head connected to a Keithley 485 autoranging picoammeter.

\section{ASSOCIATED CONTENT}

\section{Supporting Information}

The Supporting Information is available free of charge at https://pubs.acs.org/doi/10.1021/acsomega.0c02179. 
High-resolution XPS spectra of perovskite films, SEM images of perovskite films, thickness of the perovskite films, XRD pattern of perovskite films, UPS spectra of perovskite films, hysteresis index calculation, $J-V$ curves of the forward and reverse scan of PSCs, tables of PV parameters, and picture of portable plasma (PDF)

\section{AUTHOR INFORMATION}

\section{Corresponding Author}

Masoud Shekargoftar - R\&D Center for Low-Cost Plasma and Nanotechnology Surface Modifications (CEPLANT), Department of Physical Electronics, Faculty of Science, Masaryk University, 61137 Brno, Czech Republic; (1) orcid.org/00000001-6274-0942; Email: mshekargoftar@mail.muni.cz

\section{Authors}

Jan Pospisil - Faculty of Chemistry, Brno University of Technology, 61200 Brno, Czech Republic

Ján Dugácek - R\&D Center for Low-Cost Plasma and Nanotechnology Surface Modifications (CEPLANT), Department of Physical Electronics, Faculty of Science, Masaryk University, 61137 Brno, Czech Republic

Martin Weiter - Faculty of Chemistry, Brno University of Technology, 61200 Brno, Czech Republic

Tomás Homola - R\&D Center for Low-Cost Plasma and Nanotechnology Surface Modifications (CEPLANT), Department of Physical Electronics, Faculty of Science, Masaryk University, 61137 Brno, Czech Republic; (i) orcid.org/00000002-8522-6169

Complete contact information is available at:

https://pubs.acs.org/10.1021/acsomega.0c02179

\section{Notes}

The authors declare no competing financial interest.

\section{ACKNOWLEDGMENTS}

This research has been supported by GACR projects 1914770 Y and 20-29499Y funded by Czech Science Foundation and projects LM2018097 and LM2018110 funded by the Ministry of Education, Youth and Sports of the Czech Republic.

\section{REFERENCES}

(1) Chen, W.; Wang, Y.; Pang, G.; Koh, C. W.; Djurišíc, A. B.; Wu, Y.; Tu, B.; Liu, F. Z.; Chen, R.; Woo, H. Y.; et al. Conjugated Polymer-Assisted Grain Boundary Passivation for Efficient Inverted Planar Perovskite Solar Cells. Adv. Funct. Mater. 2019, 29, 1808855.

(2) Agresti, A.; Pazniak, A.; Pescetelli, S.; Di Vito, A.; Rossi, D.; Pecchia, A.; Auf der Maur, M.; Liedl, A.; Larciprete, R.; Kuznetsov, D. V.; et al. Titanium-Carbide MXenes for Work Function and Interface Engineering in Perovskite Solar Cells. Nat. Mater. 2019, 18, 12281234.

(3) Jiang, Q.; Zhao, Y.; Zhang, X.; Yang, X.; Chen, Y.; Chu, Z.; Ye, Q.; Li, X.; Yin, Z.; You, J. Surface Passivation of Perovskite Film for Efficient Solar Cells. Nat. Photonics 2019, 13, 460-466.

(4) Tavakoli, M. M.; Saliba, M.; Yadav, P.; Holzhey, P.; Hagfeldt, A.; Zakeeruddin, S. M.; Grätzel, M. Synergistic Crystal and Interface Engineering for Efficient and Stable Perovskite Photovoltaics. Adv. Energy Mater. 2019, 9, 1802646.

(5) Jena, A. K.; Kulkarni, A.; Miyasaka, T. Halide Perovskite Photovoltaics: Background, Status, and Future Prospects. Chem. Rev. 2019, 119, 3036-3103.

(6) Lin, Y.-H.; Huang, W.; Pattanasattayavong, P.; Lim, J.; Li, R.; Sakai, N.; Panidi, J.; Hong, M. J.; Ma, C.; Wei, N.; et al. Deciphering
Photocarrier Dynamics for Tuneable High-Performance PerovskiteOrganic Semiconductor Heterojunction Phototransistors. Nat. Commun. 2019, 10, 4475.

(7) Traoré, B.; Bouder, G.; Lafargue-Dit-Hauret, W.; Rocquefelte, X.; Katan, C.; Tran, F.; Kepenekian, M. Efficient and Accurate Calculation of Band Gaps of Halide Perovskites with the Tran-Blaha Modified Becke-Johnson Potential. Phys. Rev. B 2019, 99, 035139.

(8) Yu, J. C.; Badgujar, S.; Jung, E. D.; Singh, V. K.; Kim, D. W.; Gierschner, J.; Lee, E.; Kim, Y. S.; Cho, S.; Kwon, M. S.; et al. Highly Efficient and Stable Inverted Perovskite Solar Cell Obtained via Treatment by Semiconducting Chemical Additive. Adv. Mater. 2019, $31,1805554$.

(9) Chen, L.; Cao, H.; Wang, S.; Luo, Y.; Tao, T.; Sun, J.; Zhang, M. Efficient Air-Stable Perovskite Solar Cells with a (FAI)0.46(MAI)0.40(MABr)0.14(PbI2)0.86( $\mathrm{PbBr} 2) 0.14$ Active Layer Fabricated: Via a Vacuum Flash-Assisted Method under RH > 50\%. RSC Adv. 2019, 9, 10148-10154.

(10) Clark, C. P.; Voigt, B.; Aydil, E. S.; Holmes, R. J. Carrier-Gas Assisted Vapor Deposition for Highly Tunable Morphology of Halide Perovskite Thin Films. Sustainable Energy Fuels 2019, 3, 2447-2455.

(11) Xiao, X.; Bao, C.; Fang, Y.; Dai, J.; Ecker, B. R.; Wang, C.; Lin, Y.; Tang, S.; Liu, Y.; Deng, Y.; et al. Argon Plasma Treatment to Tune Perovskite Surface Composition for High Efficiency Solar Cells and Fast Photodetectors. Adv. Mater. 2018, 30, 1705176.

(12) Tavakoli, M. M.; Bi, D.; Pan, L.; Hagfeldt, A.; Zakeeruddin, S. M.; Grätzel, M. Adamantanes Enhance the Photovoltaic Performance and Operational Stability of Perovskite Solar Cells by Effective Mitigation of Interfacial Defect States. Adv. Energy Mater. 2018, 8, 1800275 .

(13) Tavakoli, M. M.; Tress, W.; Milić, J. V.; Kubicki, D.; Emsley, L.; Grätzel, M. Addition of Adamantylammonium Iodide to Hole Transport Layers Enables Highly Efficient and Electroluminescent Perovskite Solar Cells. Energy Environ. Sci. 2018, 11, 3310-3320.

(14) Si, H.; Xu, C.; Ou, Y.; Zhang, G.; Fan, W.; Xiong, Z.; Kausar, A.; Liao, Q.; Zhang, Z.; Sattar, A.; et al. Dual-Passivation of Ionic Defects for Highly Crystalline Perovskite. Nano Energy 2020, 68, 104320.

(15) Wu, Z.; Jiang, M.; Liu, Z.; Jamshaid, A.; Ono, L. K.; Qi, Y. Highly Efficient Perovskite Solar Cells Enabled by Multiple Ligand Passivation. Adv. Energy Mater. 2020, 10, 1903696.

(16) Tsai, J.-H.; Cheng, I.-C.; Hsu, C.-C.; Chen, J.-Z. DC-Pulse Atmospheric-Pressure Plasma Jet and Dielectric Barrier Discharge Surface Treatments on Fluorine-Doped Tin Oxide for Perovskite Solar Cell Application. J. Phys. D: Appl. Phys. 2018, 51, 025502.

(17) Homola, T.; Matoušek, J.; Medvecká, V.; Zahoranová, A.; Kormunda, M.; Kováčik, D.; Cernák, M. Atmospheric Pressure Diffuse Plasma in Ambient Air for ITO Surface Cleaning. Appl. Surf. Sci. 2012, 258, 7135-7139.

(18) Tsai, J.-H.; Cheng, I.-C.; Hsu, C.-C.; Chueh, C.-C.; Chen, J.-Z. Feasibility Study of Atmospheric-Pressure Dielectric Barrier Discharge Treatment on $\mathrm{CH} 3 \mathrm{NH} 3 \mathrm{PbI} 3$ Films for Inverted Planar Perovskite Solar Cells. Electrochim. Acta 2019, 293, 1-7.

(19) Zvereva, G.; Gorbanev, Y.; Graves, D. B.; Ceriani, E.; Petrovic, Z. L.; Tsai, P. A.; Verlet, J. R. R.; Jablonowski, H.; Reid, J. P.; Graham, W. G.; et al. Plasma-Liquid Interactions: A Review and Roadmap. Plasma Sources Sci. Technol. 2016, 25, 053002.

(20) Walsh, A.; Scanlon, D. O.; Chen, S.; Gong, X. G.; Wei, S.-H. Self-Regulation Mechanism for Charged Point Defects in Hybrid Halide Perovskites. Angew. Chem., Int. Ed. 2015, 54, 1791-1794.

(21) Eames, C.; Frost, J. M.; Barnes, P. R. F.; O’Regan, B. C.; Walsh, A.; Islam, M. S. Ionic Transport in Hybrid Lead Iodide Perovskite Solar Cells. Nat. Commun. 2015, 6, 7497.

(22) Kim, K. N.; Lee, S. M.; Mishra, A.; Yeom, G. Y. Atmospheric Pressure Plasmas for Surface Modification of Flexible and Printed Electronic Devices: A Review. Thin Solid Films 2016, 598, 315-334.

(23) Shekargoftar, M.; Jurmanová, J.; Homola, T. A Study on the Effect of Ambient Air Plasma Treatment on the Properties of Methylammonium Lead Halide Perovskite Films. Metals 2019, 9, 991. 
(24) Ke, C.-R.; Lewis, D. J.; Walton, A. S.; Chen, Q.; Spencer, B. F.; Mokhtar, M. Z.; Compean-Gonzalez, C. L.; O’Brien, P.; Thomas, A. G.; Flavell, W. R. Air-Stable Methylammonium Lead Iodide Perovskite Thin Films Fabricated via Aerosol-Assisted Chemical Vapor Deposition from a Pseudohalide $\mathrm{Pb}(\mathrm{SCN}) 2$ Precursor. ACS Appl. Energy Mater. 2019, 2, 6012-6022.

(25) Wang, M.; Li, B.; Yuan, J.; Huang, F.; Cao, G.; Tian, J. Repairing Defects of Halide Perovskite Films to Enhance Photovoltaic Performance. ACS Appl. Mater. Interfaces 2018, 10, 37005-37013.

(26) Zhao, P.; Kim, B. J.; Jung, H. S. Passivation in Perovskite Solar Cells: A Review. Mater. Today Energy 2018, 7, 267-286.

(27) Wang, K.; Liu, C.; Du, P.; Zheng, J.; Gong, X. Bulk Heterojunction Perovskite Hybrid Solar Cells with Large Fill Factor. Energy Environ. Sci. 2015, 8, 1245-1255.

(28) Li, Y.; Ji, L.; Liu, R.; Zhang, C.; Mak, C. H.; Zou, X.; Shen, H.H.; Leu, S.-Y.; Hsu, H.-Y. A Review on Morphology Engineering for Highly Efficient and Stable Hybrid Perovskite Solar Cells. J. Mater. Chem. A 2018, 6, 12842-12875.

(29) Shekargoftar, M.; Krumpolec, R.; Homola, T. Enhancement of Electrical Properties of Flexible ITO/PET by Atmospheric Pressure Roll-to-Roll Plasma. Mater. Sci. Semicond. Process. 2018, 75, 95-102.

(30) Hao, F.; Stoumpos, C. C.; Cao, D. H.; Chang, R. P. H.; Kanatzidis, M. G. Lead-Free Solid-State Organic-Inorganic Halide Perovskite Solar Cells. Nat. Photonics 2014, 8, 489-494.

(31) Meloni, S.; Palermo, G.; Ashari-Astani, N.; Grätzel, M.; Rothlisberger, U. Valence and Conduction Band Tuning in Halide Perovskites for Solar Cell Applications. J. Mater. Chem. A 2016, 4, 15997-16002.

(32) Shi, L. X.; Wang, Z. S.; Huang, Z.; Sha, W. E. I.; Wang, H.; Zhou, Z. The Effects of Interfacial Recombination and Injection Barrier on the Electrical Characteristics of Perovskite Solar Cells. AIP Adv. 2018, 8, 025312.

(33) Homola, T.; Ďurašová, Z.; Shekargoftar, M.; Souček, P.; Dzik, P. Optimization of Mesoporous $\mathrm{TiO} 2$ Photoanodes Prepared by Inkjet Printing and Low-Temperature Plasma Processing. Plasma Chem. Plasma Process. 2020, DOI: 10.1007/s11090-020-10086-y, , In press.

(34) Shekargoftar, M.; Dzik, P.; Durašová, Z.; Stupavská, M.; Pavliňák, D.; Homola, T. Mineralization of Flexible Mesoporous TiO2 Photoanodes Using Two Low-Temperature Dielectric Barrier Discharges in Ambient Air. Contrib. Plasma Phys. 2019, 59, 102-110. 